\title{
Phased infectivity in Heterorhabditis megidis: the effects of infection density in the parental host and filial generation
}

\author{
J.J. Ryder*, C.T. Griffin \\ Department of Biology, Institute of Bioengineering and Agroecology, National University of Ireland, Maynooth, Co. Kildare, Ireland
}

Received 18 February 2003; received in revised form 8 May 2003; accepted 8 May 2003

\begin{abstract}
Entomopathogenic nematodes can develop through two or more generations in the cadavers of killed insect hosts. Non-feeding infective juveniles from each generation emerge and may spend prolonged periods searching for a new host. The infectivity of the infective juveniles of Heterorhabditis megidis varies with time after emergence and may not reach a maximum until several weeks have passed. 'Phased infectivity' hypotheses propose that this pattern is adaptive, tending to reduce competition in new hosts. Here we provide further evidence that infectivity is phased in H. megidis. In addition, we show that the basic pattern is modified by infection density in the parental host and by filial generation. Two general patterns were observed: first, infective juveniles that developed under the least crowded conditions $\left(F_{1}\right.$ infective juveniles produced in hosts infected with 16 parent nematodes) reached maximum infectivity after only 15 days, compared to 27 or 39 days for infective juveniles that developed under more crowded conditions ( $F_{1}$ produced in hosts infected with 103 or 424 parent nematodes or $F_{2}$ infective juveniles). Second, infective juveniles had lower infectivity overall when produced under the most crowded conditions ( $F_{2}$ versus $F_{1}$; highest versus lowest infection density). We propose that while lower overall infectivity is a necessary consequence of limited resource availability during infective juvenile development, the difference in the timing of peak infectivity reflects a shift in the fitness gains associated with being maximally infective either 'early' or 'late'. $F_{1}$ infective juveniles emerge several days before $F_{2}$ infective juveniles, and we suggest that filial generation and infection density in the parental host function as indicators of the potential risk of competition within new hosts.
\end{abstract}

(C) 2003 Australian Society for Parasitology Inc. Published by Elsevier Ltd. All rights reserved.

Keywords: Heterorhabditis; Infective juvenile; Phased infectivity; Infection density; Competition

\section{Introduction}

Entomopathogenic nematodes (EPNs) of the families Steinernematidae and Heterorhabditidae are lethal parasites of insects (Poinar, 1990). The infective stage is a developmentally arrested dauer larva, or infective juvenile (IJ). After penetrating the host's cuticle, IJs kill their host by releasing toxins and insect-pathogenic bacteria into the haemocoel and resume development to sexual maturity within the cadaver. Several filial generations usually occur during the course of an infection (Wang and Bedding, 1996); each is comprised of non-infective offspring that are capable of immediate further reproduction, and a variable number of IJs. As the host's resources are depleted, an

\footnotetext{
* Corresponding author. School of Biological Sciences, Queen Mary University of London, London E1 4NS, UK. Tel.: +44-20-7882-5291; fax: +44-20-8983-0973.

E-mail address: jonryder_ie@yahoo.com (J.J. Ryder).
}

increasing proportion of the offspring in each generation develop into IJs and emerge in order to seek a new host (Ryder and Griffin, 2002).

IJs do not feed once they have emerged from their old host and must therefore accumulate sufficient lipid and carbohydrate reserves prior to emerging to survive through periods when no new hosts are available. In the long term, the consumption of these reserves is associated with a decline in the ability to infect (e.g. Menti et al., 2000). However, a number of studies have demonstrated that the proportion of IJs that infect under apparently suitable conditions initially rises with time after emergence before this decline takes place (e.g. Griffin, 1996; Campbell et al., 1999). Phased infectivity hypotheses propose that this pattern is adaptive. Hominick and Reid (1990) suggested that only a subset of the IJs that emerge from a host should be infectious immediately, with the remainder becoming infectious after a period of dormancy. This strategy is 
expected to maximise the probability that at least some IJs will infect new hosts in the event that none are present when the IJs first emerge (Fan and Hominick, 1991; Bohan and Hominick, 1995, 1996, 1997; Fenton and Hudson, 2002). Although evidence in support of this hypothesis is accumulating, Campbell et al. (1999) demonstrated that the accurate measurement of a non-infectious subset requires that specific methodological conditions be upheld. Griffin (1996) also questioned whether infection behaviour should be thought of in terms of a simple infectious/noninfectious dichotomy. Even if a truly non-infectious subset is present in a particular population (as may often be the case), the probability of any particular infectious IJ infecting a host will vary in relation to various endogenous and exogenous factors (see also Campbell et al., 1999). For example, low energy reserves may reduce the probability that an IJ will successfully locate or penetrate a host, even if it is capable of infecting.

A delayed peak in infectivity has been recorded in a number of species of EPN, suggesting that some form of phased infectivity may be relatively common (but see Campbell et al., 1999). Dempsey and Griffin (2002) have also shown that behavioural traits associated with dispersal, host finding and infectivity show phased expression in Heterorhabditis megidis (UK211 strain). Dempsey and Griffin (2002) distinguished three phases in the behaviour of H. megidis IJs: an initial dispersal phase, during which infectivity was low; an infective phase, which showed declining dispersal; and a third phase, during which the expression of all behaviours declined. This strategy may be favoured by selection if it reduces the probability of infecting nearby hosts that are already overcrowded. However, IJs that emerge relatively early during an infection cycle should have a better chance of encountering uninfected hosts than those that emerge later. Earlyemerging IJs will therefore have less to gain by delaying infectivity and should accordingly reach maximum infectivity sooner (Stuart et al., 1996). In support of this hypothesis, O'Leary et al. (1998) showed that H. megidis IJs (UK211 strain) that emerged from their hosts relatively early showed good initial host finding ability but dispersed poorly, whilst those that emerged later showed poor initial host finding ability but dispersed well.

In a previous study, we found that $H$. megidis IJs (UK211 strain) from the first filial generation $\left(F_{1}\right)$ emerged from their test host in a discrete pulse several days before the $F_{2}$ IJs emerged (Ryder and Griffin, 2002). This led us to predict that $F_{1}$ IJs will show an earlier peak in infectivity following emergence than $F_{2}$ IJs. The aim of the present study was thus to measure changes in infectivity during the period immediately following emergence in the samples of $F_{1}$ and $F_{2}$ IJs we collected previously. We also tested this prediction for IJs collected from hosts infected with three different densities of parent nematodes. The total number of early-emerging $\left(F_{1}\right)$ IJs increases with infection density in the parental host in H. megidis (Ryder and Griffin, 2002) and would be expected to have a strong effect on the adaptive value of delaying infectivity, as it should increase the risk of overcrowding within nearby hosts.

\section{Materials and methods}

\subsection{Insect and nematode cultures}

Heterorhabditis megidis (UK211 strain) were cultured in vivo using larvae of the wax moth, Galleria mellonella, as hosts (Sheffield Mealworm Company, Sheffield, UK). Infected larvae were maintained at $20{ }^{\circ} \mathrm{C}$ under continuous darkness.

\subsection{Experimental infections}

The infections that formed the basis of the present study were also used to provide the data for our previous study of density-dependent effects on fecundity and IJ production in $H$. megidis. Further details of the experimental infections can be found in Ryder and Griffin (2002). Briefly, three groups of 10 replicate dishes of $G$. mellonella larvae (nine larvae per dish) were exposed to a dose of either 50,500 or 1,000 IJs per larva. This resulted in the larvae within the three groups having mean infection densities of 16, 103 and 424 hermaphrodites per host, respectively. For each infection density, four replicates were used to monitor the development of the nematodes within their hosts (by dissection) throughout each of the three filial generations that occurred over the course of the study. The remaining six replicates were used for separate, daily collections of freshly emerged IJs in order to estimate yield and to provide the IJS for the present infectivity study.

The subsequent analysis indicated that the $F_{1}$ and $F_{2}$ IJs emerged in two discrete pulses. A peak of emergence occurred for the $F_{1}$ IJs that was separated from the peak for the $F_{2}$ IJs by a period of 3 or 4 days (the $F_{3}$ IJs emerged later still and were not studied further). This pattern occurred at each of the three parental infection densities, making it possible to harvest largely pure samples of $F_{1}$ and $F_{2}$ IJs at all three densities. IJs were harvested on every day of the emergence period for each replicate, but for the infectivity tests carried out in the present study (see below) only IJs collected on those days that corresponded to the peak of emergence for each generation were used (18-20 days after the initial infections were carried out for the $F_{1}$ IJs, and $23-$ 25 days after the initial infection for the $F_{2}$ IJs; see Ryder and Griffin, 2002, for details).

\subsection{Infectivity tests}

At each of the three parental infection densities, freshly harvested $F_{1}$ and $F_{2}$ IJs were washed by repeated sedimentation in tap water and stored at a concentration of $1,000 \mathrm{IJs}$ per $\mathrm{ml}$. Approximately $80 \mathrm{ml}$ of each replicate 
suspension (six replicate $F_{1}$ IJ harvests and six replicate $F_{2}$ $\mathrm{IJ}$ harvests for each infection density) was stored at $20{ }^{\circ} \mathrm{C}$ in continuous darkness in $9 \mathrm{~cm}$ diameter plastic food dishes (Roundstone Catering, Melksham, UK).

For each replicate, four separate infectivity tests were carried out, on days 3, 15, 27 and 39 after harvesting. Thus, each replicate IJ suspension was removed from storage and the concentration of viable IJs was determined from the mean of five $0.1 \mathrm{ml}$ samples. A sample of approximately 1 $\mathrm{ml}$ was taken from the suspension, adjusted (diluted) to a concentration of $200 \mathrm{IJs}$ per $\mathrm{ml}$ and ten $0.5 \mathrm{ml}$ sub-samples were added to 10 individual $5 \mathrm{~cm}$ diameter Petri dishes lined with filter paper (the suspension was returned to storage until the next infectivity test). A single G. mellonella larva was introduced into each dish and incubated for $24 \mathrm{~h}$. After that time, each larva was removed, washed under tap water, returned individually to a clean dish, and incubated for a further 7 days. Insects were dissected and the number of adult first generation nematodes present was counted. Thus, infectivity was measured as the mean number of IJs that successfully established (developed to adulthood) in a single G. mellonella larva after a 24-h exposure period. Ten G. mellonella larvae were dissected per infectivity test, and each test was replicated six times (i.e. one infectivity test per replicate IJ suspension) on each of the 4 days on which sampling was carried out. The same procedure was followed for the $F_{1}$ and $F_{2}$ IJs at each of the three parental infection densities.

\subsection{Statistical analysis}

The experimental design had three factors: parental infection density $(16,103$ or 423 hermaphrodites per host), filial generation $\left(F_{1}\right.$ or $\left.F_{2}\right)$ and time after emergence $(3,15$, 27 or 39 days). Data were analysed using GLM in Minitab (release 13.1). After ln-transformation, data did not deviate significantly from normality (Kolmogorov-Smirnov test). Error variances did not differ significantly across groups (Levene's test).

\section{Results}

In order to measure changes in infectivity with time after emergence, each replicate IJ suspension (dish) was sampled on four separate occasions (on days 3, 15, 27 and 39). Therefore, to control for the possibility of a dish effect, a GLM Anova was constructed with 'parental infection density', 'filial generation', and 'time after emergence' as fixed factors, and 'dish' as a random factor nested within 'infection density'. There was no significant effect of 'dish' on mean infectivity and no interaction with 'generation', and so the 'dish' term was dropped from the model.

The final model is shown in Table 1 (ln-transformed data). There were highly significant effects of all three factors on mean infectivity. There were also significant
Table 1

Three-way analysis of variance (GLM) for the effect of time after emergence, filial generation and parental infection density on mean infectivity

\begin{tabular}{lrrr}
\hline Source & d.f. & $F$ ratio & \multicolumn{1}{l}{$P$} \\
\hline Time & 3 & 61.16 & $<0.001$ \\
Generation & 1 & 98.14 & $<0.001$ \\
Density & 2 & 10.81 & $<0.001$ \\
Time $\times$ generation & 3 & 8.10 & $<0.001$ \\
Time $\times$ density & 6 & 5.50 & $<0.001$ \\
Generation $\times$ density & 2 & 0.86 & 0.427 \\
Time $\times$ generation $\times$ density & 6 & 5.67 & $<0.001$ \\
Error & 120 & & \\
\hline
\end{tabular}

two-way interactions between time after emergence and both infection density and filial generation, and a significant three-way interaction. Fig. 1 shows how mean infectivity changed with time for the $F_{1}$ and $F_{2}$ IJs for each of the three parental infection densities. Infectivity initially increased with time after emergence in all treatments. However, parental infection density and filial generation both affected this general pattern.

Table 2 shows post hoc multiple comparison (Tukey) tests for differences among the levels of each factor. $F_{2}$ IJs generally showed significantly lower infectivity than $F_{1}$ IJs on any given day, for all three infection densities. The only exception to this pattern was that $F_{2}$ IJs showed significantly higher infectivity than $F_{1}$ IJs 39 days after emergence when derived from the lowest infection density.

There were also significant differences among days within each infection density and generation. $F_{1}$ IJs that emerged from host infected with 16 parent nematodes increased significantly in infectivity from day 3 to 15 and declined significantly thereafter. In contrast, $F_{1}$ IJs derived from the upper two infection densities and $F_{2}$ IJs derived from all three densities continued to increase significantly in infectivity from day 3 until day 27 or 39 . Thus, the point after emergence at which maximum infectivity occurred differed between parental infection densities.

High parental infection density was also associated with a lower level of infectivity for the $F_{2}$ IJs. The main effect of infection density on the $F_{1}$ IJs was a shift in the point at which maximum infectivity occurred (there were no significant differences in infectivity for the $F_{1}$ IJs between the intermediate and highest infection densities, which showed the same basic changes over time). However, for the $F_{2}$ IJs infectivity dropped to a significantly lower level by day 39 at the highest parental infection density, compared to the lowest or intermediate densities.

\section{Discussion}

This study provides further support for the conclusion of Griffin (1996) and Dempsey and Griffin (2002) that infectivity increases with time after emergence in $H$. megidis 
$\mathbf{A}$ 16 hermaphrodites per host

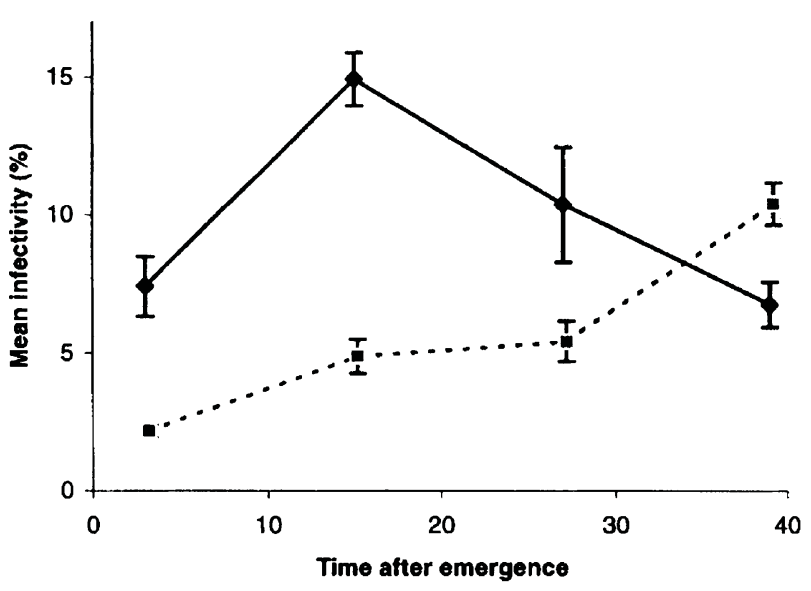

103 hermaphrodites per host

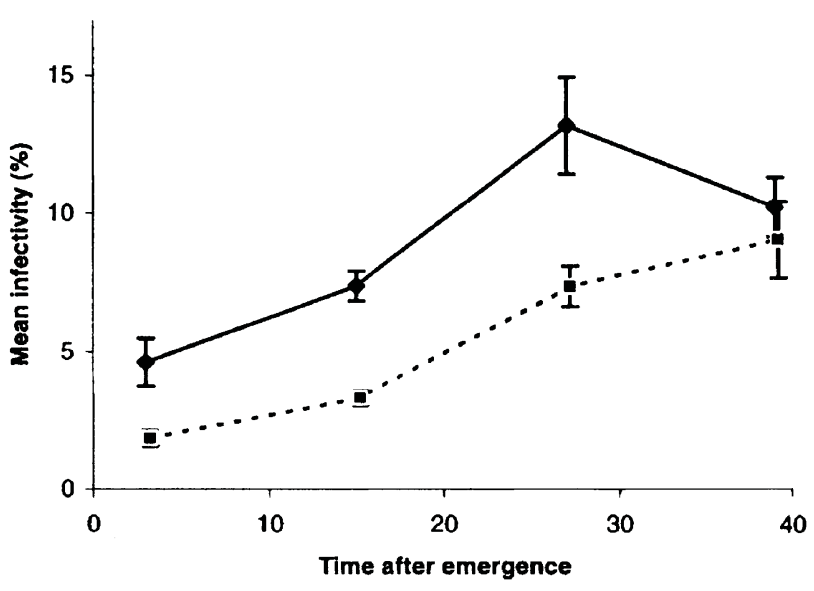

C 424 hermaphrodites per host

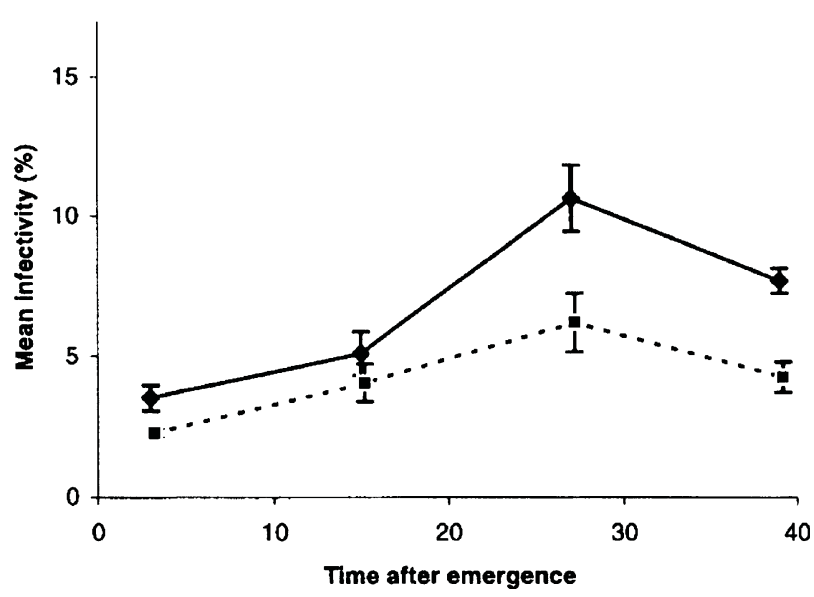

Fig. 1. The effect of parental infection density and filial generation on the relationship between time after emergence (days) and infectivity (percentage of infective juveniles that successfully established in test hosts after 24 h): A, 16 hermaphrodites per host; B, 103 hermaphrodites per host; C, 424 hermaphrodites per host. Means are presented \pm standard errors: $(-\bullet-)$ $F_{1}$ infective juveniles; (- - - - - ) $F_{2}$ infective juveniles.
(UK211 strain). We detected a general increase in infectivity after the first test (on day 3) for both filial generations and all three parental infection densities. However, both of these factors modified the basic form of the relationship. Filial generation per se had a strong effect, with $F_{1}$ IJs tending to be more infective than $F_{2}$ IJs at any given point after emergence. Parental infection density affected the time at which maximum infectivity occurred, with $F_{1}$ IJs showing an earlier peak in infectivity when derived from the lowest parental infection density. Infectivity was also lower after 39 days for $F_{2}$ IJs that were derived from the highest infection density than for $F_{2}$ IJs from the lowest or intermediate densities.

Much of the discussion of 'phased infectivity' in Steinernema species has focused on changes in the size of a non-infectious subset of IJs, which will not infect hosts even under suitable conditions. We concentrated instead on estimating variation in infectivity, defined as the proportion of IJs that infected a single host within a limited (24 h) period. These two concepts of phased infectivity are not mutually exclusive, but demonstration of a non-infectious subset requires a different experimental approach (in particular, that IJs are supplied with an excess of host individuals; Campbell et al., 1999). It is therefore possible that the between-treatment differences we detected in temporal variation in infectivity overlook underlying changes in the size of a non-infectious subset. Such a finding would not contradict the changes we detected in infectivity, but it would be interesting to test whether and to what extent the two factors that were the focus of this study-infection density in the original host and filial generation-affected the number of non-infectious IJs.

The results presented here, and those previously published by Griffin (1996) and Dempsey and Griffin (2002), are consistent with a pattern of phased infectivity in $H$. megidis. However, our data also indicate a degree of plasticity that may function to maximise the probability of successful transmission when competition for new hosts is high. When the original hosts harboured a mean of 16 hermaphrodites each, the $F_{1}$ IJs, which were the first to emerge, showed an earlier peak in infectivity than the $F_{2}$ IJs, which emerged several days later. $F_{1}$ IJs were thus more likely to infect hosts encountered soon after emerging if parental infection density was low. Data presented previously for this strain indicate that the total number of $F_{1}$ IJs that emerge from a single host originally infected with 16 hermaphrodites may be over 500-fold less than the total number of $F_{2}$ IJs that emerge from the same host (Ryder and Griffin, 2002). As a consequence, when parental infection density is low $F_{1}$ IJs should have more to gain by reaching maximum infectivity early on because there will be less competition within hosts encountered soon after emerging (see also Stuart et al., 1996).

Clearly, simply being in the first cohort of IJs to emerge may mean that competition for nearby hosts will be lower, which may in itself tend to favour an 'infect early' strategy. 
Table 2

Post hoc tests for differences among the levels of each factor: generation ( $F_{1}$ and $F_{2}$ infective juveniles), time (days) and density (16, 103,424 hermaphrodites per host)

\begin{tabular}{|c|c|c|c|c|c|c|c|c|c|c|}
\hline \multicolumn{3}{|c|}{ Among generations ${ }^{\mathrm{a}}$} & \multicolumn{4}{|c|}{ Among days ${ }^{\mathrm{b}}$} & \multicolumn{4}{|c|}{ Among densities ${ }^{c}$} \\
\hline Day & Density & $\left(F_{1}-F_{2}\right)$ & Day & Density & $F_{1}$ & $F_{2}$ & Density & Day & $F_{1}$ & $F_{2}$ \\
\hline 3 & 16 & $*$ & 3 & 16 & $\mathrm{a}$ & $\mathrm{a}$ & 16 & 3 & $\mathrm{a}$ & $\mathrm{a}$ \\
\hline 15 & & $*$ & 15 & & $\mathrm{~b}$ & $\mathrm{~b}$ & 103 & & $a b$ & $\mathrm{a}$ \\
\hline 27 & & n.s. & 27 & & $a b$ & $\mathrm{~b}$ & 424 & & $\mathrm{~b}$ & $\mathrm{a}$ \\
\hline 39 & & $*$ & 39 & & $\mathrm{a}$ & $\mathrm{c}$ & 16 & 15 & $\mathrm{a}$ & $\mathrm{a}$ \\
\hline 15 & & $*$ & 15 & & $\mathrm{~b}$ & $\mathrm{~b}$ & 424 & & $\mathrm{~b}$ & $\mathrm{a}$ \\
\hline 27 & & $*$ & 27 & & $\mathrm{c}$ & $\mathrm{c}$ & 16 & 27 & $\mathrm{a}$ & $\mathrm{a}$ \\
\hline 39 & & n.s. & 39 & & $\mathrm{bc}$ & $\mathrm{c}$ & 103 & & $\mathrm{a}$ & $\mathrm{a}$ \\
\hline 3 & 424 & $*$ & 3 & 424 & $\mathrm{a}$ & $\mathrm{a}$ & 424 & & $\mathrm{a}$ & a \\
\hline 15 & & n.s. & 15 & & $\mathrm{ac}$ & $a b$ & 16 & 39 & $\mathrm{a}$ & $\mathrm{a}$ \\
\hline 27 & & $*$ & 27 & & $\mathrm{~b}$ & $\mathrm{~b}$ & 103 & & $\mathrm{~b}$ & $\mathrm{a}$ \\
\hline 39 & & $*$ & 39 & & $\mathrm{bc}$ & $\mathrm{b}$ & 424 & & $a b$ & $\mathrm{~b}$ \\
\hline
\end{tabular}

a One-way Anova comparing $F_{1}$ and $F_{2}$ IJs on each day and at each density: $*=P<0.05$.

b Tukey HSD comparisons among days within each level of density and generation; significant at $P<0.05$ where letter codes differ.

c Tukey HSD comparisons among densities within each level of time and generation; significant at $P<0.05$ where letter codes differ.

But the data presented here and previously suggest that competition will also be high amongst $F_{1}$ IJs when infection density is high in the original host. When hosts were infected with 103 hermaphrodites, we found previously that approximately 10 times as many $F_{1}$ IJs emerged in comparison with the number that emerged at 16 hermaphrodites per host, whilst the number of $F_{2}$ IJs was reduced to well below the number of $F_{1}$ IJs (Ryder and Griffin, 2002). $F_{1}$ IJs that developed at both of the upper two infection densities (103 and 424 hermaphrodites per host) also showed a delayed peak in infectivity. Taken together, these data suggest that filial generation and infection density in the original host may serve as indicators of the potential risk of competition within hosts that are encountered soon after emerging. The IJs in each generation may therefore be primed at some stage during development to become maximally infective either 'early' or 'late', depending on the potential risk.

O'Leary et al. (1998) showed that early-emerging $H$. megidis IJs (UK211 strain) favoured rapid host location (and presumably infection) instead of dispersal, whilst lateemerging IJs were initially poor at finding hosts and instead tended to disperse. O'Leary et al. also suggested that a density-dependent cue within the host may be responsible for mediating which of these two different behavioural strategies are expressed in the phenotype. Our data provide further support for this conclusion. However, it is important to stress that the differences we detected in infectivity occurred under the particular conditions imposed by our infectivity tests, which used a single host. In each test, an implicit component of the observed infection behaviour was therefore the response of a proportion of the IJs towards an already infected and possibly less suitable host (as only one host was available). How this behaviour would be modified by the presence of additional hosts remains unclear.
The 'adaptive' explanation presented above rests on an important assumption. If delayed infectivity is to be selected for in the manner suggested above, there must be substantial costs associated with reproducing within a high-density infection. Hermaphrodites developing in hosts that are overcrowded are considerably less fecund than those developing in less crowded conditions (Ryder and Griffin, 2002). However, if IJs 'choose' not to infect (i.e. demonstrate a lower probability of infecting) any hosts encountered soon after emerging in order to avoiding potential overcrowding, might not the risk that they will fail to find another host outweigh any potential gain in fecundity? Estimating the associated fitness costs and benefits of such a decision is beyond the scope of this paper. However, it is important to note that a proportion of the IJs that emerge from a host will be full siblings produced by self-fertilising hermaphrodites. There may therefore be some kin selection to avoid overcrowding nearby hosts if they are likely to contain sibling IJs already, as this may reduce inclusive fitness.

Although our results support the adaptive hypothesis, the data also suggest an alternative explanation: that the changes we observed in the temporal pattern of infectivity were the product of a physiological constraint associated with overcrowding. There was a consistent tendency for IJs that emerged from host cadavers that were in a more advanced state of depletion (i.e. $F_{2}$ IJs or IJs emerging from more heavily infected hosts) to show delayed and/or reduced infectivity. Our previously reported data show that the parents of these IJs produced far fewer offspring than parent nematodes that developed in better conditions (i.e. earlier in the course of an infection or under less competition). It is therefore possible that their offspring accumulated lower levels of energy reserves and that this explains the effect on temporal variation in infectivity. 
However, this hypothesis is perhaps only to likely apply to those IJs that show evidence of reduced rather than delayed infectivity - in particular, to $F_{2}$ IJs that developed at the highest parental infection density. It is more difficult to explain why IJs with low energy reserves would still attain relatively high infectivity, albeit at a later stage-particularly if that were to mean initially spending more time dispersing, as Dempsey and Griffin's (2002) data suggest. It seems unlikely, therefore, that the differences we observed in infectivity can be fully explained in terms of a physiological constraint, although this may at least in part explain the reduced pattern that occurred at the highest parental infection density.

All species of EPN rely on IJs to ensure transmission. The future reproductive success of those IJs will be heavily influenced by competition within new hosts, which greatly reduces fecundity. The phased infectivity hypothesis suggests a mechanism that may enable EPNs to minimise the degree to which this constraint reduces transmission success. This study shows that the basic shape of the relationship between time after emergence and infectivity in $H$. megidis depends on infection density in the original host and on a (filial) generational effect. Although several studies of phased infectivity in EPNs have been conducted, none has yet focused on the effect of these two factors explicitly. Nevertheless, temporal variation in infectivity in each filial generation appears to respond to the degree of competition in the parental host in a manner that may maximise fitness. Our data suggest that future studies of infectivity in $H$. megidis and other EPNs may benefit from taking these two factors into account. Differences in basic culturing and infection protocols (such as the dose of IJs used to infect in in vivo culture, or the time at which IJs are harvested) may otherwise have a substantial effect on the results.

\section{Acknowledgements}

This work was supported by the Higher Education Authority, Ireland, under its Programme for Research in Third Level Institutions. We are very grateful to Joan Hunter and Gemma Lynch for help with insect dissections and to Catherine Hurley for statistical advice. Two referees provided comments that improved the manuscript.

\section{References}

Bohan, D., Hominick, W., 1995. Examination of the Steinernema feltiae (Site 76 strain) infection interaction with the Galleria mellonella host, using an infection model. Parasitology 111, 617-625.

Bohan, D., Hominick, W., 1996. Investigations on the presence of an infectious proportion amongst the populations of Steinernema feltiae (Site 76 strain) infective stage. Parasitology 112, 113-118.

Bohan, D., Hominick, W., 1997. Long-term dynamics of infectiousness within the infective-stage pool of the entomopathogenic nematode Steinernema feltiae (Site 76 strain) Filipjev. Parasitology 114, 301-308.

Campbell, J.F., Koppenhöfer, A.M., Kaya, H.K., Chinnasri, B., 1999. Are there temporarily non-infectious dauer stages in entomopathogenic nematode populations: a test of the phased infectivity hypothesis. Parasitology 118, 499-508.

Dempsey, C.M., Griffin, C.T., 2002. Phased activity in Heterorhabditis megidis infective juveniles. Parasitology 124, 605-613.

Fan, X., Hominick, W.M., 1991. Effects of low storage temperature on survival and infectivity of two Steinernema species (Nematoda: Steinernematidae). Revue de Nématologie 14, 407-412.

Fenton, A., Hudson, P.J., 2002. Optimal infection strategies: should macroparasites hedge their bets? Oikos 96, 92-101.

Griffin, C.T., 1996. Effects of prior storage conditions on the infectivity of Heterorhabditis sp. (Nematoda: Heterorhabditidae). Fundam. Appl. Nematol. 19, 95-102.

Hominick, W.M., Reid, A.P., 1990. Behaviour of infective juveniles. In: Gaugler, R., Kaya, H.K. (Eds.), Entomopathogenic Nematodes in Biological Control, CRC Press, Boca Raton, FL, pp. 327-345.

Menti, H., Wright, D.J., Perry, N.P., 2000. Infectivity of populations of the entomopathogenic nematodes Steinernema feltiae and Heterorhabditis megidis in relation to temperature, age and lipid content. Nematology 2 , 515-521.

O'Leary, S.A., Stack, C.M., Chubb, M.A., Burnell, A.M., 1998. The effect of day of emergence from the insect cadaver on the behavior and environmental tolerances of infective juveniles of the entomopathogenic nematode Heterorhabditis megidis (strain UK211). J. Parasitol. $84,665-672$.

Poinar, G.O. Jr, 1990. Taxonomy and biology of the Steinernematidae and Heterorhabditidae. In: Gaugler, R., Kaya, H.K. (Eds.), Entomopathogenic Nematodes in Biological Control, CRC Press, Boca Raton, FL, pp. $23-61$.

Ryder, J.J., Griffin, C.T., 2002. Density-dependent fecundity and infective juvenile production in the entomopathogenic nematode, Heterorhabditis megidis. Parasitology 125, 83-92.

Stuart, R.J., Lewis, E.E., Gaugler, R., 1996. Selection alters the pattern of emergence from the host cadaver in the entomopathogenic nematode Steinernema glaseri. Parasitology 113, 183-189.

Wang, J., Bedding, R.A., 1996. Population development of Heterorhabditis bacteriophora and Steinernema carpocapsae in the larvae of Galleria mellonella. Fundam. Appl. Nematol. 19, 363-367. 